\title{
Muslimisches Recht - Zwischen liberaler Reform und reaktionärem Fundamentalismus ${ }^{\mathrm{r}}$
}

\author{
»Unsere kulturelle Aufgabe ist es jetzt, den Stier bei den Hörnern zu packen und \\ Selbstkritik zu üben, bevor wir das Bild kritisieren, das andere sich von uns \\ machen, und sei es auch ein absichtlich verzerrtes". \\ Fouad Zakariya in: Laicite ou islamisme - les arabes a l'heure du choix, I99I \\ "Es gibt keine arabische Rasse und keine arabische Nation. Es gibt eine Sakral- \\ sprache, die des Qur'an, die von Herrschern benutzt wird, damit die Leute an der \\ Entdeckung ibrer eigenen Identität verhindert werden."
}

Kateb Yacine, berberischer algerischer Schriftsteller, I989

Es gibt bei dem europäischen Blick auf die islamischen Gesellschaften des Mittleren Ostens zwei Schablonen, durch die Muslime wahrgenommen werden: Die erste ist die abwertend rassistische. Die vermittelten Bilder der »Arabischen Straße« bestehen aus einem wütenden Mob, der israelische und amerikanische Fahnen verbrennt und sich dabei selbst geißelt. Auf der anderen Seite wird gerade in akademischen Kreisen ein romantisiertes Bild reproduziert. Der soziale Zusammenhalt, die Gastfreundschaft und die exotische Tradition des Orients werden angeführt. Eine im Westen angeblich verloren gegangene Authentizität und Identität der gesellschaftlichen Ordnung scheint im Mittleren Osten noch zu bestehen. In dieser Weise präsentierten sich auch die auf der Frankfurter Buchmesse 2004 vertretenen Diktaturen der Arabischen Liga: Kinderbücher, schnitzende arabische Männer und verschleierte arabische Frauen, die Europäerinnen Henna-Tattoos aufmalen. Ebenfalls eine starke Strömung der deutschen Orientalistik fördert die romantischen Projektionen des säkularisierten Europas auf den Orient. So veröffentlichte die deutsche Orientalistin Annemarie Schimmel, die I94 I in Berlin promovierte, Bücher wie »Kleine Paradiese. Blumen und Gärten im Islam «, ${ }^{2}$ während sie Verständnis für die Todesdrohung der iranischen Fatwa gegen Salman Rushdi äußerte. ${ }^{3}$

Eine wissenschaftliche, an den realen Entwicklungen und Auseinandersetzungen der arabischen Welt orientierte Debatte über den Zusammenhang von Islam und Menschenrechten muss sich von diesen beiden fatalen Sichtweisen frei machen. Die Debatte über die potenzielle Geltungskraft von Menschenrechten in islamischen Gesellschaften wird sich zwischen den Polen eines humanistischen Universalismus und eines kulturellen Relativismus wiederfinden.

Warum zu bestimmten Zeiten wissenschaftliche Auseinandersetzungen stattfinden, hängt mit den Problemlagen einer Zeit zusammen, so dass sich Wissenschaft nicht erlauben kann, ihren eigenen politischen Kontext in den Erkenntnisprozess nicht mit einzubeziehen. ${ }^{4}$

In der rechtlichen Debatte stellt sich die Frage: Können dieselben, in den kapitalistisch-bürgerlichen Gesellschaften des Westens errungenen bzw. postulierten Menschenrechte auch in islamisch geprägten Gesellschaftsformationen gelten?

In seinem Beitrag zu Islam und Menschenrechten macht Timo Tohidipur dem ihm

I Dieser Artikel ist als Antwort zu Timo Tohidipur in der Kritischen Justiz 2004, S. 305 ff., konzipiert, welcher selbst auf Calalettin Kartal, KJ 2003, S. 382 , antwortet.

2 Schimmel, Annemarie, Kleine Paradiese. Blumen und Gärten im Islam, Freiburg $200 \mathrm{I}$.

3 Zur Debatte um Schimmel anlässlich der Verleihung des Friedenspreises des Deutschen Buchhandels: Der richtige Preis? Neue Debatte um Annemarie Schimmel, nzz 07. I0. I 995; Tilman Spengler (Hrsg.), Wenn ich Roman Herzog wär ...; Schwarzer, Alice, Die falsche Toleranz, Köln 2002, S. I 3.

4 Vgl. Günther, Frieder, Vom Staat her denken, München 2004, S. 9 ff. 
vorhergegangenen Artikel von Celalettin Kartal die Vorwürfe, das Ergebnis seiner Untersuchung vorwegzunehmen, die Moderne unzulässiger Weise zum Maßstab aller menschlichen Gesellschaften zu machen, ein lineares Geschichtsbild zu vertreten und so, methodisch bedingt, keine neutrale, das andere in seiner Eigentlichkeit anerkennende Analyse der islamischen Gesellschaftsordnung zu treffen. Tohidipurs Interesse scheint es zu sein, einer Diffamierung des Koran entgegenzutreten. Diese bestehe darin, dass Kartal fordert, auch Moslems müssten akzeptieren, dass der Koran

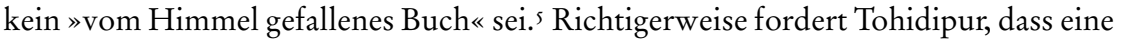
rechtsvergleichende Untersuchung nicht lediglich unreflektierte Bewertungen vornehmen darf, sondern zunächst unvoreingenommen versucht, »kennen zu lernen «, wobei der Erkenntnisgegenstand das eigene und das betrachtete Rechtsmodell sind. Methodisch setzt er sich von Richtig und Falsch als Leitkriterien ab, um diese dann aber lediglich durch ein bloßes So und Anders zu ersetzen. Durch diese Beschränkung der Analyse auf reine Deskription stellt er sich somit in einen kulturrelativistischen Kontext. Der Kulturrelativismus entstand um 1900 in Abgrenzung zum Ethnozentrismus, der die eigene Ethnie als das Zentrum aller Dinge bewertet und alle anderen Kulturen im Hinblick auf die eigene Weltanschauung beurteilt. Gleichzeitig richtet er sich aber auch gegen einen Universalismus, der die Existenz einer allumfassenden Ethik einfordert. ${ }^{6}$

Von diesem methodischen Ansatz aus zeigt Tohidipur das Bild eines Islam, welcher in der Umma (Gemeinschaft der gläubigen Moslems) die sozialen Rechte des Subjekts gewährleistet, und er stellt den Iran so dar, dass man den Eindruck gewinnt, er sei eine islamische Musterdemokratie zwischen göttlichem und menschlichem Recht. Er gibt also ein bestimmtes Selbstverständnis des Islam als monotheistischer Offenbarungsreligion wieder.

Das Setzen von Werten, die Richtig und Falsch bestimmen, ist sicherlich bei einem Rechtsvergleich eine Verschleierung des eigenen Bewertungsstandpunktes. Die bloße Unterscheidung in So und Anders läuft aber Gefahr, das Vorgefundene zu ontologisieren und statische Identitäten zu konstruieren, indem sie das Selbstbild des Erkenntnisobjektes bloß reproduziert.7 Verschieden geartete Gesellschaftsformationen stehen immer im Bezug zueinander. Ihre geschichtliche Dynamik erhalten sie durch ihre inneren und äußeren Widersprüche. Die Ontologisierung von Identitäten als angeblich authentisch und eigen blendet diesen geschichtlichen und dialektischen Entwicklungsprozess aus.

Die Angst vor der Förderung eurozentristischer Debatten darf nicht dazu führen, dass die begriffliche Schärfe in der Analyse verloren geht. Insofern ist das Abtun der Moderne durch Tohipidur mit dem Argument, diese sei ja gar keine feste wissenschaftliche Figur, lediglich ein tu quoque (du auch) Argument, das an dem primären Erkenntnisgegenstand vorbei geht. Dass die aufgeklärte Moderne ein widersprüchlicher Prozess ist, der auch die industrielle Vernichtung von Menschen ermöglicht hat und insofern selbst kritikwürdig ist, enthebt wiederum nicht »den Islam« als Herrschaftsideologie und -form von Kritik.

Im Folgenden soll gezeigt werden, wie sich im Mittleren Osten die Staatsbildung nach dem Modell so genannter hydraulischer Gesellschaften im Unterschied zum Westen

5 Tohidipur, KJ 2004 , S. 320.

6 Vertreter des Kulturrelativismus: Edward Sapir/Judith T. Irvine, The Psychology of Culture, 2002; des weiteren: Alfred Kroeber/Margaret Mead/Ruth Benedict, Zu Islam und Kulturrelativismus; Kamguian, Azam, The Lethal Combination of Tribalism Islam \& Cultural Relativism, Vortrag 19.01. 2003 in Stockholm, http://www.secularislam.org/women/tribalism.htm. (Alle Links im Internet wurden das letzte Mal am 28.0I. 2005 auf Aktualität überprüft.)

7 Vgl. Würth, Anna, Dialog mit dem Islam als Konflikt-Prävention?, Bad Honnef/Berlin 2003, S. $9 \mathrm{ff}$. 
vollzogen hat, so dass daraus Herrschaftsordnungen entstanden sind, denen aus sich selbst heraus eine Anerkennung subjektiver Rechte der Unterworfenen fremd ist. Der Islam wird hierbei als eine historische Erscheinung berücksichtigt und eingeordnet. Sodann soll untersucht werden, inwieweit es eine geschlossene islamische Rechtstradition gibt, die als Basis verschiedener muslimischer Rechtsordnungen dient. Der entscheidende Unterschied zu Tohidipur wird es sein, nicht nur ein Islamverständnis wiederzugeben, sondern die wesentlichen Pole darzustellen, zwischen denen sich heute die Rechtsdiskussionen in muslimischen Gesellschaften bewegen. Hierbei wird eindeutig Position für eine säkulare Entwicklung bezogen, da, wie sich zeigen wird, islamische Argumentationen heute der Entfaltung eines individualrechtlichen Denkens im Mittleren Osten entgegenstehen.

Wenn von islamischem Recht gesprochen wird, so wird im Allgemeinen die Ordnung der Scharia gemeint. Hierbei leitet schon der Begriff »islamisches « Recht fehl, da er sehr unterschiedliche konkrete Rechtsordnungen mehrerer Staaten vereinheitlicht. Die Rechtsordnungen des Mittleren Ostens stellen, auch wenn sie alle den Islam zumindest als eine Rechtsquelle zugrunde legen, kein einheitliches System dar. Eine unmittelbare Anwendung der Scharia erfolgt nur in sehr wenigen Ländern (z. B. Iran, Sudan, Saudi Arabien und Nordnigeria). Auch bieten die islamischen Schriften der Sunna und des Korans kein systematisch geschlossenes System. Nach der Präsentation des Koran durch den Propheten und Herrscher Mohammed entwickelten die islamischen Rechtsgelehrten (die fiqhs) nach dessen Tod schon vier verschiedene islamische Rechtsschulen. ${ }^{8}$ Der Koran selber brauchte wohl 200 Jahre, um zu seiner heutigen Fassung zu gelangen. ${ }^{9} \mathrm{Da}$ sich der Islam seit seiner Entstehung und gewaltsamen Durchsetzung von Nordafrika bis nach Asien, die durch den Kolonialismus und die gewalttätige Eroberung der Welt durch Europa zu einem Ende kam, ${ }^{\circ}$ nicht immer gleich dargestellt hat, sind auch heutige Selbstbilder des Islam nicht für sein ewiges Wesen zu halten.

Des Weiteren impliziert der Begriff islamisches Recht, dass dieses Recht direkt aus dem Willen Gottes abgeleitet wird. Die Rechtsordnungen islamisch geprägter Gesellschaften sind aber alle von Menschen, nämlich Muslimen, die sich als Anhänger des Islam verstehen, errichtet worden. Von daher erscheint der Begriff »muslimisches Recht « als angebrachter, da er sich auf die Subjekte, welche die jeweiligen Rechtsordnungen aufgebaut haben, bezieht. ${ }^{\text {I }}$

Der Islam sah sich bei seiner Durchsetzung mit unterschiedlichen regionalen Gesellschaften konfrontiert. ${ }^{22}$ Die folgende Argumentation wird sich in mehreren Schritten dem Thema annähern: A. Gesellschaftliche Strukturierung orientalischer Despotien im Vergleich zu bürgerlichen Gesellschaften, B. Grundlagen islamischer Rechtsideologie, C. Überblick über die Standpunkte der aktuellen Rechtsdebatte der Arabischen Staaten.

8 U. A.: i An-Naim, Abdullah, >The Best of Times and sthe Worst of Times Rights in Islamic Societies, Muslim World Journal of Human Rights, Volume I (2004), http://www.bepress.com/mwjhr/volı/issi/art5; Tibi, Bassam, Die Entwestlichung des Rechts! - Das Hudud-Strafrecht der islamischen Shariá, in: Lüderssen, Aufgeklärte Kriminalpolitik oder Kampf gegen das Böse, BadenBaden I998, S. 2 I.

9 S. Raddatz, Hans-Peter, Verbotene Liebe - Die deutsche Islamwissenschaft kuscht vor radikalen Glaubenswächtern, Rheinischer Merkur, Nr. 50, 09. I 2. 2004, http://www.merkur.de/aktuell/ku/ku_०4500I. html.

ıo Vgl. Raddatz, Hans-Peter, Von Allah zum Terror? Der Djihad und die Deformierung des Westens, München 2002.

I I Kathleen McNeil, Shari'a, Traditional Muslim Jurisprudence, Muslim Laws: A Plea for Replacing the term Islamic Law, http://www.whrnet.org/fundamentalisms/docs/issue-sharia0407.html.

I 2 Sarkohi, Faraj, Islam als Religion und Staatsideologie, nzz 03. 02. 200I; http://www.nzz.ch/2001/02/03/li/ page-article7 I IRP.html. 

zu bürgerlichen Gesellschaften

Rechtssysteme sind keine willkürlich geschaffenen Systeme, sondern sie sind historische Erscheinungen, die sich in bestimmten sozioökonomischen Strukturen herausbilden. Die Schaffung verbindlicher rechtlicher Ordnungen hat dabei die Funktion, einen erträglichen Status quo aufrechtzuerhalten, der eine berechenbare Dynamik der Fortentwicklung ermöglicht. Recht ist dabei die Einsicht, dass eine Gesellschaft übergeordnete Instanzen schaffen muss, wenn sie die in ihr bestehenden Antagonismen nicht mehr einer Selbstregulation überlassen kann. So erhält Staatlichkeit eine doppelte Funktion als neutraler Regulator gesellschaftlicher Konflikte und als Instanz zur Absicherung gegebener Herrschafts- und Machtverhältnisse. ${ }^{13}$ Von daher ist zum Verständnis von Rechtssystemen und -ideologien ein Verständnis des sozioökonomischen Umfeldes entscheidend, da so verständlich wird, in welcher Form das Recht seine Funktion gegenüber den Mitgliedern einer Gesellschaft wahrnimmt.

Um die unterschiedliche Entwicklung des Westens und des Mittleren Ostens verständlich zu machen, wird hier auf die Theorie der asiatischen Produktionsweise und der so genannten hydraulischen Gesellschaft, welche das Entstehen der orientalischen Despotien bedingt, zurückgegriffen. Das Konzept der hydraulischen Gesellschaft wurde von Karl A. Wittfogel, der in Weimar durch das Frankfurter Institut für Sozialforschung sozialisiert wurde und sich im Kalten Krieg zu einem erbitterten Gegner der Sowjetunion entwickelte, unter Bezugnahme auf Weber, Marx und Montesquieu entwickelt.

Ausgehend davon, dass menschliche Gesellschaften sich in einer Auseinandersetzung mit ihren natürlichen Bedingungen entwickeln, erkennt Wittfogel die Unterschiede der Staatsbildung von Europa und dem Orient. In einer lebensfeindlichen Umgebung erscheint die Abhängigkeit von dem lokal begrenzt verfügbaren Rohstoff Wasser höher als in Gebieten mit wesentlich mehr Niederschlag. Von daher ergibt sich die Notwendigkeit monumentaler Bauprojekte, die auf einer zentralen Planung beruhen. Orientalische Gesellschaften sind deshalb im Gegensatz zu westlichen Gesellschaften, welchen Hydroagrikulturen mit regelmäßigem Niederschlag zugrunde liegen, hydraulische Gesellschaften.

Hydraulisch bedeutet, dass zentral angelegte Wasserverteilungsanlagen errichtet werden müssen, um eine dauerhafte Struktur zu schaffen, welche die Grundversorgung menschlicher Gemeinschaften absichert. Es entsteht »die unbedingte Notwendigkeit einer sparsamen und gemeinschaftlichen Verwendung des Wassers (...) im Orient, wo die Zivilisation zu niedrig und die territoriale Ausdehnung zu groß war, um freiwillige Assoziationen ins Leben zu rufen, (was) das Eingreifen einer zentralisierten Staatsgewalt erforderlich machte «. ${ }^{14}$ Die zentrale Koordination der gemeinschaftlichen Infrastrukturprojekte wird die erste Aufgabe des Staates. Es entsteht eine existenzielle Abhängigkeit der einzelnen Dorfgemeinschaften von der Zentralgewalt. Mit den notwendigen zentralistischen Bau- und Organisationsmethoden entwickelt der hydraulische Staat gegenüber der Gesellschaft ein enormes Aneignungspotential, welches sich auf ein umfangreiches Berufsbeamtentum stützen kann. Die so entstehenden Organisationsfähigkeiten kann der Herrscher nach eigener Willkür einsetzen, wodurch nicht nur Infrastrukturgroßprojekte, sondern auch großangelegte Bauten zur Repräsentation von Macht möglich werden. So ergibt sich ein geschichtliches

I 3 Agnoli, Johannes, Die Transformation der Demokratie, Freiburg 1990, S. $33 \mathrm{ff}$.

I4 Wittfogel, Karl A., Die Orientalische Despotie - Eine vergleichende Untersuchung totaler Macht, Berlin I 977 , S. 462. 
Entwicklungsmodell, das sich von einem deterministischen Phasenmodell nach europäischen Maßstäben, welches davon ausgeht, dass gesellschaftliche Entwicklungen zwangsläufig die gleichen Stadien wie europäisch geprägte Länder durchlaufen, verabschiedet hat. ${ }^{\text {is }}$ Ein dezentralistischer Feudalismus, der einzelnen Kommunen eine politisch relevante Autonomie gewährleistete, hat sich im Orient nie herausgebildet. Der Feudalismus, als einem absolutistischen Herrscher entgegengesetzte starke Herrschaftsklasse, bildete in Europa eine wesentliche Quelle des später entstehenden bürgerlichen Föderalismus. ${ }^{16}$ Die regionalen Repräsentanten der Macht im Orient hatten dagegen mehr die Funktion von Steuereintreibern als die autonomer Fürsten. ${ }^{17}$

Einen Feudalismus, der Institutionen entwickelte, um eigene Interessen gegenüber der zentralen Macht zu vertreten und durchzusetzen, hat es im Orient nie gegeben. »Und es war der verstreute Zustand der orientalischen Bevölkerung in selbstgenügsamen Dörfern (verbunden mit kleiner Landwirtschaft und Hausindustrie), der, wiederum Marx zufolge, den fortdauernden Bestand des Staates ermöglichte. ${ }^{18}$ Größere Städte entstanden nur an für Handel und Warenumschlag geeigneten Plätzen oder um den Sitz des Herrschers herum. Da die Städte eine in hohem Maße auf das Zentrum hin bezogene Funktion besaßen, konnte sich auch hier, viel schwerer als in Europa, eine autonome, bürgerliche Ständegesellschaft herausbilden.

Dem sich aus dieser Struktur erhebenden Staat liegt eine dem Westen gegenüber andere Eigentumsstruktur zu Grunde. Es ist politisch starkes von schwachem Eigentum zu unterscheiden. Grundsätzlich beherrscht die staatliche Bürokratie das Eigentum an den strategisch entscheidenden Produktionsmitteln, insbesondere dem Ackerland. Städte sind dabei die administrativen und militärischen Stützpunkte des Regenten. Zwar entwickelte sich auch ein vom Staat unabhängiges Eigentum, dessen Inhaber hatten aber, wenn sie nicht selbst schon mit dem Staat verwoben waren, nicht die Möglichkeit, sich zu politisch wirkungsmächtigen, unabhängigen Zentren zusammenzuschließen. Gegenüber der organisatorischen Macht der besitzenden zentralen Bürokratie konnten sich zwar Privateigentümer entwickeln, aber - häufig mitbedingt durch ein zentral gelenktes Erbrecht - diese konnten keine organisierte Macht bilden. Dem orientalischen Absolutismus gelang ein Grad an Zentralisierung, den die absolutistischen Herrscher Europas nie erreichten. ${ }^{19}$ Die herrschende und besitzende Klasse ist hier die hydraulische Bürokratie. ${ }^{\circ}$ Durch ihre totale Macht über den Einzelnen sind die grundsätzlichen Herrschaftsstrukturen des Orients in einem wesentlich höheren Maße statisch als in der nicht hydraulischen Welt. Zwar kommt es immer wieder zu einem Wechsel der Cliquen in der Führungsebene, die grundsätzliche Form der Herrschaft bleibt aber bestehen.

Diese Konzeption von Wittfogel hat einen breiten Anklang in der wissenschaftlichen Debatte gefunden. Ein wesentlicher Einwand gegen seine Theorie war, er würde den altgriechischen Propaganda-Mythos vom guten Europa und dem bösen Asien wieder beleben. ${ }^{21}$ Persönlich war es Wittfogels primäres Interesse, sich dem Totalitarismus entgegenzustellen, so dass sein Buch in der Tat auch als eine politische Abhandlung aus

Is Witzens, Udo, Kritik der Thesen Karl A. Wittfogels über den »Hydraulischen Despotismus«, Heidelberg 2002 , S. 66.

I6 Wittfogel (Fn. I4), S. Iо I ff.

I7 Wittfogel (Fn. I4), S. 466.

I 8 Wittfogel (Fn. I4), S. 462.

i9 Wittfogel (Fn. I4), S. i s 5 ff.

20 Dass Staatseigentum in Orientalischen Despotien nicht mit Volkseigentum durch Verstaatlichung, das im Interesse der Bürger verwaltet wird, zu verwechseln ist, wird in der aktuellen Debatte immer wieder nicht in ausreichendem Maße wahrgenommen. So z. B. exemplarisch: Klein, Naomi, Blätter für deutsche und Internationale Politik I/os.

2I Toynbee, The American Political Science Review, March 1958, 52:I, S. I95 ff. 
der Zeit des Kalten Krieges zu verstehen ist. Die Grundlagen hat Wittfogel bereits I 938 in dem Beitrag »Die Theorie der orientalischen Gesellschaft«, Zeitschrift für Sozialforschung I 938, 90 ff. gelegt. Jedoch stellt er in seiner Analyse auf den Ansatz ab, die konkrete menschliche Eigentumsorganisation und die Konfrontation mit der den Menschen umgebenden Ökologie als entscheidende Faktoren für den Aufbau einer Gesellschaft zu setzen. Insofern werden in Wittfogels Orientalischer Despotie in erster Linie unterschiedliche Vergesellschaftungsformen beschrieben. ${ }^{22}$ Es wird nicht auf ontologische Stereotypen abgestellt, sondern die Bedingungen totaler Herrschaft in außereuropäischen Gesellschaftsformationen werden offen gelegt.

Positiv bezieht sich auf Wittfogel z. B. Samuel Huntington ${ }^{23}$ in seinem »Clash of Civilisations «. ${ }^{24}$ Huntington, der Anfang der 9oer Jahre versuchte, die Konfliktlinien des $2 \mathrm{I}$. Jahrhunderts zu beschreiben, bleibt der bloßen Deskription verhaftet, und es gelingt ihm kaum, die internen Bedingungen und Bewegungsfaktoren für das Aufeinanderprallen der von ihm benannten Kulturkreise zu erfassen.

Für die Frage nach der möglichen Implementierung und Geltungskraft von Menschenrechten in der muslimischen Welt muss im Folgenden geklärt werden, in welcher Form das Recht seine Funktion gegenüber den Rechtsunterworfenen in der hydraulischen Welt ausfüllt.

Die politische Schwäche des privaten Eigentums führte zu einer vom Westen unterschiedlichen Ausbildung des Verhältnisses von Subjekt und Staat, was sich insbesondere in der unterschiedlichen Bedeutung von Öffentlichkeit in Orient und Okzident zeigt. Wie schon Marx feststellte, hat eine abstrakte Trennung des Individuums in eine private und in eine öffentliche Sphäre in nicht westlichen Kulturkreisen nicht im gleichen Maße stattgefunden. ${ }^{25}$ Diese ist aber die Vorraussetzung dafür, dass der Einzelne für seine Interessen in einer Form politisch einsteht, die es ihm ermöglicht, Rechte einzufordern, zu formulieren und Kooperationen zu schaffen, aus denen eine politische Stärke gegenüber der Herrschaft entsteht. So sind auch Menschenrechte nicht gottgegeben oder vorstaatlich, auch wenn sie in historischen Phasen derart begründet werden, sondern sie entwickeln sich aus einem Prozess historischer Kämpfe, die den Menschen in das Zentrum menschlichen Denkens setzen, zwischen Herrschaft und Unterworfenen und zwischen Mensch und Natur. ${ }^{26}$

Die zentrale Instanz bürgerlicher Staatlichkeit dafür ist die Öffentlichkeit. Eine solche gibt es auch in den Gesellschaften des Mittleren Ostens. Hier hat sie aber eine zu den westlichen Gesellschaftsformationen gegensätzliche Funktion und Genese.

In der Ideologie der bürgerlichen Gesellschaft ist die Öffentlichkeit die Instanz, in der das souveräne Volk die maßgeblichen Entscheidungen über sich selbst und seine Staatlichkeit trifft. Durch Öffentlichkeit werden Machtentscheidungen staatlicher Körperschaften legitimiert und kontrolliert. In der Öffentlichkeit wird zumindest über die Form der Repräsentanz der verschiedenen Interessen der Gesellschaft bestimmt. Das Programm der Aufklärung forderte eine Repräsentanz des Volkes im Gegensatz zur bloßen Repräsentanz der Macht in der Öffentlichkeit. Nach

22 Krader, Larence, Asiatische Produktionsweise, in: Haug (Hrsg.), Historisch-Kritisches Wörterbuch des Marxismus, Band I, Berlin I994, S.635.

23 Huntington, Samuel P., Kampf der Kulturen, München 2002, S. 98.

24 Der in Deutschland viel besprochene, aber wohl wenig gelesene Huntington argumentiert aus einer konservativ isolationistischen Perspektive. Seine These von den »bloody borders of islam « erregte vor allem Anstoß. Zu Huntington: Menzel, Ulrich, The West against the Rest: Samuel Huntingtons Rekonstruktion des Westens, in: Peripherie Nr. 68 1997, S.6r; Zum Verhältnis Wittfogel/Huntington: Witzens (Fn. I 5), S. 7 I ff.

25 Massenzio, Marcello, Ethnologie, in: Haug (Hrsg.), Historisch-Kritisches Wörterbuch des Marxismus, Band 3, Berlin 1994, S. 927; Marx, Karl, MEW 42, 383 ff.

26 Vgl. Bloch, Ernst, Naturrecht und menschliche Würde, Frankfurt 1978; Agnoli, Johannes, Subversive Theorie, Freiburg 1996. 
Vertragstheoretikern wie Rousseau kann zwar der Wille der Bevölkerung einen Fürsten bestimmen, dieser steht aber nicht außerhalb des Rechts, so dass der Staat selber keinen Eigenwert in dem Sinne besitzt, dass er sich von der Idee her gegen die Interessen der ihm Unterworfenen stellen kann und darf.

Die Voraussetzung eines für die Regierung des freien und rechtlich gleichen Subjekts über sich selbst derart zentralen Forums sind bestimmte Eigenschaften und Fähigkeiten des einzelnen Bürgers. Er benötigt einen hohen Bildungsstandard, um in der Lage zu sein, reflektierte Bewertungen von Entscheidungen treffen zu können. Um sich seine Autonomie zu wahren, ist des Weiteren eine gewisse soziale Homogenität von Nöten, die nach den Vorstellungen der Theoretiker der Aufklärung (Locke, Hobbes, Rousseau) durch das Recht auf individuelles Eigentum gewährleistet werden soll. ${ }^{27}$ Real hat ein derartiges Ideal von Öffentlichkeit in den kapitalistischen Gesellschaften des Westens wohl nie existiert. ${ }^{28}$ Das Verhältnis von Citoyen und Bourgeois ist wesentlich fragiler und komplexer ausgestaltet, als dass real alle Bürger einer antagonistisch aufgebauten Gesellschaft die faktische und soziale Möglichkeit haben, die ihnen zugesprochenen Möglichkeiten und Rollen real auszufüllen.

Im Kampf gegen Absolutismus, Klerus und Feudalität entwickelte dieser politische Anspruch aber eine reale Wirkungsmacht in der Strukturierung von Staat und Gesellschaft. In dieser Auseinandersetzung ergaben sich auch Anknüpfungspunkte der aufsteigenden bürgerlichen Ordnung an den ausgebildeten Dezentralismus feudaler Ordnung und der relativen Entscheidungsautonomie innerhalb der feudalen Klasse.

Derartige Voraussetzungen für Öffentlichkeit gibt es in den islamischen Gesellschaften des Mittleren Ostens nicht. Nicht der griechische Versammlungsort des Demos ist das Ideal von Öffentlichkeit nahöstlicher Gesellschaften, sondern der Bazar. ${ }^{29}$ Orte für größere Menschenansammlungen sind im Wesentlichen Moscheen.

Auf dem Bazar werden Waren zur Schau gestellt und nicht politische Entscheidungen getroffen. In der Öffentlichkeit des Orients kommt es nicht zur Repräsentanz einer Interessengruppe oder der Herrschaft gegenüber dem anderen, um Entscheidungen $\mathrm{zu}$ treffen oder sie legitimieren zu lassen, sondern der eigene Besitz und der eigene Status werden zur Schau gestellt.

Der Staat war über die Kontrolle der natürlichen Rohstoffe im Besitz von starkem Eigentum, welches die (Selbst)unterwerfung des Untertans forderte. Demgegenüber können die Untertanen keine politische Autonomie entwickeln, da sie schon mit einer bloßen Abkehr von der Herrschaft ihre eigene Reproduktion gefährden würden. Notwendig, um in der Entscheidung des Herrschers positiv berücksichtigt zu werden, war immer die Zurschaustellung von Loyalität. Der allgemein zugängliche Raum war von daher auch nie ein Raum, in dem politische Entscheidungen getroffen wurden. Es wurde auch nicht der Anspruch darauf formuliert. Das sich zur Schau stellende Subjekt selbst wiederum wird durch die Präsentation seiner selbst der Kontrolle der Gemeinschaft unterworfen, welche von der Herrschaft total abhängig ist. Althergebracht ist Öffentlichkeit in den islamischen Gesellschaften ein Kontrollmodus für das Subjekt und nicht der Ort, in dem es sich selbst bestimmt oder behauptet.

27 Siehe zu den verschiedenen Konzeptionen von Öffentlichkeit grundlegend Arendt, Hanna, vita activa oder vom täglichen Leben, München 2002; Habermas, Jürgen, Strukturwandel der Öffentlichkeit, Frankfurt I962.

28 Pohrt, Wolfgang, Der Geheimagent der Unzufriedenheit - Balzac: Rückblick auf die Moderne, Berlin I 990, S. 8 I ff.

29 Arendt (Fn. 27), S. I90. 
Das in der Öffentlichkeit zur Schau Gestellte ist das Ich-Ideal einer gesellschaftlichen Funktion, nicht das selbstbewusste und auch selbstkritische Individuum. Hier greift auch »das Kernelement der islamischen Tradition, die Pflichtenlehre « $3^{\circ}$ ein, von welcher Tohidipur spricht. Ihr ist ein individualistisches Rechtsverständnis fremd. Das Fehlen eines organisierten und wirkungsmächtigen Bürgertums, das sich das Verständnis von individuellen Rechten und Rechtspersonen zueigen gemacht hätte, war in den I950er und I960er Jahren z. B. für die kommunistischen Parteien des Mittleren Ostens als säkulare politische Kräfte in ihren strategischen Entscheidungen ein Hauptgesichtspunkt, nicht auf eine alleinige Übernahme der Macht zu drängen. ${ }^{3 r}$ Fraglich erscheint bei Zugrundelegung dieser These vom Zusammenhang von Öffentlichkeit und Subjektkonstitution, inwieweit sich trotzdem im Mittleren Osten die Möglichkeit ergeben hat oder ergeben kann, dass das Subjekt Rechte für sich einfordern kann.

\section{B. Grundlagen islamischer Rechtsideologie}

Im Folgenden ist zu klären, inwieweit das Modell der Orientalischen Despotie auch heute zum Verständnis muslimischer Gesellschaften und Rechtssysteme beitragen kann und welche Rolle der Islam als jüngster Offenbarungsreligion in diesem Konzept spielt.

Keines der Länder des Mittleren Ostens ist demokratisch verfasst. Insoweit es Parlamente gibt, sind diese einem Herrscher oder einer Instanz wie dem iranischen Wächterrat - über den noch zu sprechen sein wird - unterworfen. Nach dem Arab Human Development Report $2003^{32}$ der UN wurden alle arabischen Staaten in den Skalen zu Lebenserwartung, Prokopfeinkommen, Analphabetismus und schulische Standards als niedrig eingestuft. In allen diesen Ländern existiert eine ineffektive bürokratische Struktur. Trotz des Kapitals, das durch die Ausbeutung natürlicher Ressourcen in die Region floss, blieb das Einkommen der Bevölkerungsmehrheit relativ niedrig, während sich die Herrschaftscliquen, wie das Königshaus Saud, bereicherten. Zugleich sind diese Länder mit einer Bevölkerungsexplosion und einer einhergehenden Arbeitslosigkeit konfrontiert, die von der Bürokratie nicht mehr aufgefangen werden kann. ${ }^{33}$

Die Ökonomien des Mittleren Ostens sind weniger auf Warenproduktion denn auf das Ausbeuten von Quellen und die Verteilung des Gewinns unter den Herrschaftseliten ausgerichtet. Die grundsätzlich von Wittfogel beschriebene Situation besteht bei einer gewissen Ausdifferenzierung je nach Land also noch heute.

Technische Modernisierungsschübe und die Entwicklung in der Türkei haben zu Beginn und Mitte des 20. Jahrhunderts in vielen muslimischen Ländern zwar zu der Herausbildung eines kleinen Bürgertums geführt. Dieses konnte sich aber nie zu einer das System revolutionierenden Gruppe zusammenschließen. Im Mittleren Osten kam es zu einer Übernahme westlicher Ideen, die antikolonial gewendet wurden, was seinen Ausdruck in einem lokalen Nationalismus oder in einem Panarabismus fand. Der Panarabismus versuchte, islamische Begriffe wie den der Umma, der Gemein-

30 Tohidipur, KJ 2004, S. 3 I 3.

3 I S. dazu z. B. für den Irak Farouk-Sluggett, Marion/Sluggett, Peter, Der Irak seit 1958 - Von der Revolution zur Diktatur, Frankfurt I99i, S. 72 ff., i I 7 ff.

32 Arab Human Development Report 2003 (2003) Published for the United Nations Development Programme (UNDP). New York: Oxford University Press.

33 Rubin, Barry, The tragedy of the middle east, Cambridge 2002, S. 6. 
schaft aller Moslems (Umma al islam), säkular als Gemeinschaft der Araber umzudeuten (al Umma al-arabiyya), um dabei gleichzeitig den Anschluss an die religiösen Bevölkerungsmassen zu behalten. ${ }^{34}$

Umma ist als ein universaler Rechtsbegriff zu verstehen. Er bezeichnet ursprünglich die Gemeinschaft aller gläubigen Muslime, wobei er sich nicht an den Grenzen souveräner Staaten orientiert. Unterworfen sind die Mitglieder der Umma unter die Jurisprudenz Gottes, nicht die einer menschlichen Ordnung. Aus Sicht der Umma al islam sind auch nicht-muslimische Araber Ausländer bzw. Ungläubige. ${ }^{35}$ Vergleichbar ist die Konzeption von Umma daher eher mit dem ius sanguini der deutschen Rechtstradition denn mit dem ius sol der französischen und angelsächsischen. Das die Gemeinschaft verbindende Element ist nicht verwaltungstechnisch, sondern kulturell definiert. Daher steht sowohl in der deutschen als auch in der muslimischen Tradition die Gemeinschaft und nicht das Individuum im Mittelpunkt. ${ }^{6}$ Bei der Umma handelt es sich nicht nur um einen abstrakt spirituellen Begriff, sondern die der Umma Zugeordneten unterfallen der Entscheidungshoheit religiöser Würdentrager. Dies wurde für den Westen insbesondere durch den Fall Salman Rushdies demonstriert, gegen welchen von Ayatollah Khomeini eine Fatwa mit Todesurteil erlassen wurde, obwohl Rushdie nicht einmal in einem muslimischen Land lebte. ${ }^{37}$

Mit dem Scheitern des Überfalls der arabischen Armeen auf Israel im Sechs-TageKrieg wurde der sich im Panarabismus andeutende Säkularismus im Mittleren Osten aus islamistischer Sicht in Diskredit gebracht..$^{8}$ Daraufhin stand der Slogan »Der Islam ist die Lösung « (al-Islam huwa al-hall), der von der institutionell orientierten Moslembruderschaft aus Ägypten geprägt wurde, symbolisch für die islamistische Offensive einer inneren Reformation der arabischen Welt.

Diese Reformationsbewegung ging nicht nur von staatlichen Instanzen aus, sondern insbesondere von der islamistischen Opposition, die sich seit den zwanziger Jahren erfolgreich organisierte (so z. B. Muslimbruderschaft, Hisbollah oder Hamas) und sich über ein aufgebautes Netzwerk karitativer Einrichtungen verankerte. Dem gegenüber zeichnet sich sowohl die Opposition säkularer Kräfte als auch die von einem Reformislam durch eine tiefe organisatorische Schwäche aus. ${ }^{39}$

Das Ende des Ost-West-Konfliktes, welcher aus der Sicht der moslemischen Welt vor allem ein interner Konflikt des Westens war, machte die strukturelle Krise des Mittleren Ostens offenbar. So beschreibt der amerikanische Experte für den Mittleren Osten, Barry Rubin, die Situation nach 1989: »What was taking place in the Middle East, then, was not much a confrontation of civilisations as something far simpler and quite common in world history: the determination of elites and systems to survive >oppositionists < efforts to seize power for themselfes, and reactionary hatred and fear of what others called >progress $\ll «^{\circ}{ }^{\circ}$

34 Milson, Menahem, Reform vs. Islamism in the arab world today; www.memri.de, special dispatch i 5 . Sept., 2004 .

35 Tohidipur spricht Bassam Tibi die wissenschaftliche Qualität ab (s. KJ 2004, 306, Fn. 4), ohne sich auch nur mit einem seiner Argumente auseinanderzusetzen. Auch wenn ein etwas selbstverliebter Schreibstil abschrecken mag, so entkräftet dies nicht die gebrachten Fakten und Argumente. Also seien diese hier zitiert: Tibi, Bassam, Fundamentalismus im Islam. Eine Gefahr für den Weltfrieden?, Darmstadt 2000, S. $60 \mathrm{ff}$.

36 Küntzel, Matthias, Jihad und Judenhass - über den neuen antijüdischen Krieg, Freiburg 2002, S. 34; Lewis, Bernard, Treibt sie ins Meer! Die Geschichte des Antisemitismus, Frankfurt 1987, S. I73.

37 Tibi, Bassam, Der neue Totalitarismus - »Heiliger Krieg« und westliche Sicherheit, Darmstadt 2004, S. $70 \mathrm{ff}$.

38 Ebd.

39 Rubin (Fn. 33); Milson (Fn. 34), S. I I; Würth (Fn. 7), S. 46 ff.

40 Rubin (Fn. 33), S. Io. 
Hier setzt die heutige Rechtsdiskussion im Mittleren Osten an. ${ }^{4 \mathrm{I}}$ Die Frage nach einer generellen Vereinbarkeit von »dem Islam» mit Menschenrechten ist falsch gestellt. Menschenrechtsverletzungen der arabischen Regime sind nicht allein auf den Islam zurückzuführen, sondern auch auf das allgemeine despotische Herrschaftsinteresse. Die muslimische Rechtsordnung der Scharia wird von politischen Akteuren und Intellektuellen in verschiedener Weise als argumentativer Bezugspunkt genutzt. Die Rechtsprechung auf Basis der islamischen Scharia fußt nicht auf einem kodifizierten Recht, weil die Scharia als göttliches Gesetz nur interpretativ durch Deutung des Korantextes und des Hadith (Überlieferung des Propheten) ausgelegt werden kann. Es gibt kein islamisches Rechtsbuch, wie etwa das BGB, namens Scharia. ${ }^{42}$ Hier sollen vier generelle Positionen unterschieden werden: a. islamistische Oppositionsbewegungen mit wahabitischer und salafistischer Ideologie, die Menschenrechte als westliches Konzept verwerfen, b. der Islam als staatstragende Ideologie, der als Legitimationsgrundlage einer authentischen muslimischen Ordnung gesehen wird, dem im konkreten ein Menschenrechtsdialog aufgezwungen ist, deren Akteure ihre Macht aber mit Gewalt verteidigen, c. ein Reformislam, der aus der Scharia Menschenrechte entwickeln will, und d. Positionen, die eine Durchsetzung der Menschenrechte nur gegen die Schariaordnung als möglich ansehen.

\section{a) Islamisten: "Der Islam ist die Lösung"}

Vor dem Aufstieg des Fundamentalismus seit den I $920 e r n$ wurde die Scharia nicht als Staatsrecht verstanden. Außer einer Funktion der post-eventum-Legitimation politischer Handlungen der Herrscher diente sie als Zivilrecht auf der kommunitären Ebene der Madhahib (konfessionelle Rechtsschulen) und war vorwiegend auf Erbschafts- und Eheangelegenheiten beschränkt. ${ }^{43}$ Mit dem Scheitern säkularer Ideen in der arabischen Welt setzte ein Prozess ein, der von dem Historiker Hobsbawn als die Erfindung von Traditionen bezeichnet wurde. ${ }^{44}$ So wurde der strafrechtliche Teil der Scharia, sog. Hudud-Strafen, in den Vordergrund gerückt. Hierbei erscheint die Schariaordnung primär als ein Disziplinierungsmittel und angeblich als authentische Quelle muslimischer Ordnung. Es wird eine so nie existente Ordnung in die Vergangenheit projiziert, die angeblich bestand, bevor Europa seinen blutigen Siegeszug um die Welt antrat und den Kolonialismus der arabischen Herrscher ${ }^{45}$ durch den eigenen überholte.

Ein Beispiel für diese Erfindung von Tradition ist Nigeria, wo die Scharia im Laufe des Jahres 2000 als Strafrecht-zivilrechtlich galt sie in vielen Gliedstaaten schon davor-in I 2 von 36 Bundesstaaten im Norden des Landes eingeführt wurde. ${ }^{46}$

Für die arme und ungebildete Bevölkerung des Nordens verknüpfte sich die Einführung der Scharia mit der Hoffnung auf Gerechtigkeit, der Eindämmung von

4I Für den genaueren Aufbau von Scharia, Sunna, Koran und den Haddiths sei auf den sehr informativen Artikel von Calalettin Kartal (KJ 2003, S. 382) verwiesen, der sich mehr auf den ideologischen und politischen Kontext muslimischen Rechts konzentriert.

42 Tibi (Fn. 8), S. 2 I; Raddatz (Fn. 9).

43 Tibi ebd.

44 Vgl. Hobsbawn, Eric/Ranger, Tarrence (Hrsg.), The Invention of Traditions, Cambridge I983.

45 Dazu Raddatz (Fn. 9).

46 Zum Recht in Nigeria s.: Becker, Heike, Voreheliche Sexualität und traditionelles Recht in Nordnamibia, in: Peripherie Nr.65/66 1997, S. I 57 ff.; L Adamu, Fatima, Haushaltsstrategien, Frauen und ShariaGerichtshöfe in Nord-Nigeria, in: Peripherie Nr. 952004. 
wie die Amputationen wegen Diebstahls oder Prügelstrafen wurden ohne weitreichende nationale oder internationale Proteste vollstreckt. Große Aufmerksamkeit haben dagegen zwei Urteile gegen Frauen erhalten, die wegen außerehelichen Geschlechtsverkehrs - sie waren nach einer Scheidung schwanger geworden - zum Tod durch Steinigung verurteilt wurden. Diese Urteile wurden in der Folge aufgehoben.

Obwohl die Scharia-Gerichte explizit in einem Land mit circa $50 \%$ Christen nur für Muslime gelten sollen, hat deren Einführung direkte Auswirkung auf »Ungläubige«. Geschlechtertrennung beiöffentlichen Verkehrsmitteln betrifft Frauen aller Religionsgruppen; das Verbotvon Alkoholverkauf und Prostitutiongefährdet die wirtschaftliche Basis zahlreicher Migranten aus dem Süden. ${ }^{77}$ Die Folgen der Einführung der Scharia in Nordnigeria für Moslems und Nicht-Moslems zeigt auch, dass die Erhebung des Islam zur alles umfassenden Ideologie die Trennung von ziviler und öffentlicher Sphäre für das Subjekt negiert und somit einen totalitären Anspruch hat. $4^{8}$

Der Islam drang über die transsaharischen Handelswege im Laufe von Jahrhunderten in Nigeria ein. Zu Beginn des I 9. Jahrhunderts kam es zum Jihad Usman dan Fodios, der die Islamisierung weiter vorantrieb und in dessen Folge das erste Kalifat errichtet wurde. Dieses blieb aber politisch schwach, was eine Kolonialisierung durch die Briten erleichterte. Diese ließen das Scharia-System teils bestehen, wobei sie den Schariagerichten verboten, Todesstrafen zu verhängen, Amputationen durchzuführen und zu steinigen.

Zufolge der neuen, in Nord-Nigeria sehr populären islamischen Geschichtsschreibung war die Region seit langem im Grundsatz islamisch, und Usman dan Fodio etablierte einen wahrhaft islamischen Staat, in dem die Scharia so »vollständig « implementiert war wie nirgendwo sonst außerhalb der arabischen Welt. Die Wiedereinführung des Sharia-Strafrechts heute sei damit ein Ausdruck einer vollständigen Entkolonialisierung. 49

Diese Erfindung von Traditionen geht einher und füllt sich inhaltlich mit einer Abgrenzung gegenüber dem Feind, der im gottlosen Westen gesehen wird. Eine Kernfrage für die islamistische Opposition ist es, ob ihr Hauptfeind in den eigenen, nicht islamistischen Despoten des Mittleren Ostens zu suchen ist oder im Westen und in Israel. Khaled Abou El Fadl, ein islamischer Reformer in den Vereinigten Staaten, beschreibt, dass zeitgenössische islamistische Intellektuelle den Islam nur »in der Antithese zum Westen konstruieren statt der Menschheit eine moralische Vision zu geben. In der Welt, die sich diese Gruppen aufbauen, gibt es keinen Islam; in dieser Welt gibt es nur den Widerstand gegen den Westen «. . $^{\circ}$

Ein Beispiel dafür aus einer Diskussion mit dem saudischen Scheich Khaled AlKhlewi über die Gleichheit von Frauen und Männern: »Some of those who demand (women's rights) transfer the experience of European and Western society to here.... My dears, sense and logic reject this, let alone religious law. Allah did not create such a thing as complete equality. ... If in a certain workplace women have no choice but to mix with men, this is prohibited according to Islamic religious law. Therefore, when the Western woman left her home and met with men in all places, in factories,

47 Harneit-Sievers, Axel, Jihad vs. Miss World: Politik, Religion und Geschichte in Nigeria, Vortrag vor dem Zentrum Moderner Orient, Berlin, 22. September 2003, http://www.uni-kassel.de/fb Io/frieden/regionen/ Nigeria/harneit.html

48 Zum totalitären Charakter des Islamismus: Tibi, Bassam, Der neue Totalitarismus - \#eiliger Krieg « und westliche Sicherheit, Darmstadt 2004.

49 S. Harneit-Sievers (Fn. 47).

so Zitiert nach Emran Qureshi I. Brief. Der Briefwechsel wurde zwischen Juni und August 2004 geführt und ist erstmals erschienen in der Frankfurter Rundschau vom 4. Oktober 2004. http://www.qantara.de/ webcom/show_article.php/_c-323/_nr-8/i.html. 
workshops, in laundromats, in kitchens, in all places, within a few months the results were bitter: rape cases, abortions, and even in marriage, there was a horrifying degree of failure because the woman went out to work. «s

\section{b) Die Despoten: "Das freie Wablsystem ist für unser Land nicht anwendbar «s}

Durch die derartig organisierte islamistische Opposition gerieten die despotischen Regime des Mittleren Ostens unter Druck, so dass sie in der Gesetzgebung nachgaben, um ihren Herrschaftsanspruch zu sichern und ideologisch zu legitimieren. Z.B. wurden unter Mubarak in Ägypten islamistische Gruppen, die seinen Herrschaftsanspruch in Frage stellten, zunächst brutal niedergedrückt. Soweit sie ihre Feinderklärung aber nur nach Außen richteten, konnten sie eine zunehmende Geltung der Scharia in der nationalen Gesetzgebung durchsetzen. So wurde 1984 abgeschafft, dass ein Mann seine Ehefrau um Erlaubnis fragen muss, bevor er eine zweite heiraten darf.53 Seit den I990ern ist es in Ägypten unter Bezug auf die Scharia möglich, Muslime, die sich verdächtig gemacht haben, vom Glauben abgefallen zu sein, Zwangszuscheiden. So wurde der Linguistik-Professor Hamid Abu Zaid auf höchstrichterlichen Beschluss von seiner Frau geschieden, da es verboten ist, dass eine Muslimin mit einem Ungläubigen verheiratet ist. ${ }^{54}$

Gleichzeitig stehen diese Regime aber auch durch eine Internationalisierung des Menschenrechtsdiskurses unter Druck, so dass es zu einer Verstaatlichung des Menschenrechtsdiskurses kam. Dies fand in manchen Ländern in den r990ern seinen Ausdruck in der Einrichtung verschiedener Stellen innerhalb von Ministerien. 55 Inwieweit dies Wirkung zeigt oder ob es sich bloß um eine Technik zur Ruhigstellung der liberalen Opposition und einer gegen die UN gerichteten Nationalisierung der Menschenrechtspolitik handelt, bleibt abzuwarten. ${ }^{56}$

Die Regime versuchen also, einen Spagat zwischen Verteidigung einer islamischen Authentizität und den an sie gestellten Ansprüchen von Außen und den liberalen Intellektuellen zu vollführen. Die Scharia ist dabei das Aushängeschild der Verteidigung islamischer Werte gegenüber und für den Islamismus.

So verteidigt exemplarisch der saudische Kleriker Said Al-Qahtani die auf der Scharia basierenden Körperstrafen: »We ask [the Westerners]: >What kind of people do you defend? $<$ Islamic law defends the innocent and stops the criminal. In the West, it's the other way around. They defend the criminal and sacrifice and persecute the innocent. When the Islamic law chops off a hand, it does so because this hand is criminal ... This is a medical principle these days. If a patient had gangrene in his finger, shouldn't we chop it off? Is this barbarism? No. Why do we chop it off? Not to be cruel to the patient, but out of mercy for his whole body. « 57 Zugleich wird der Vorwurf doppelter Standards gegen den Westen erhoben, indem auf Israel verwiesen wird: »Interviewer: On the other hand those who claim that the application of Islamic punitive law is barbarism, and I'm talking about our enemies - see what they are doing to people?... Said Al-Qahtani: Now the Palestinians are being killed daily, and the world is silent.

5I MemriTV, 6/29/2004, Clip No. I37, http://www.memritv.org/Transcript.asp?PI=I 37 .

52 König Fahd von Saudi Arabien, zitiert nach: Huntington, Samuel, The Clash of Civilisations, Foreign Affairs Summer 1993, S. 49.

53 Serauky, Eberhard, Im Namen Allahs. Der Terrorismus im nahen Osten, Berlin 2000, S. 68.

54 Tibi (Fn. 35), S. 105.

55 Z. B. Marokko, Indonesien, Jemen. Seit 2003 auch Ägypten und Saudi Arabien, welches unter dem Eindruck des Irakkrieges auch das erste Mal Kommunalwahlen durchführen ließ.

56 Dazu: Würth (Fn. 7), S. 50.

57 MemriTV, I I/24/2004, Clip No.385, http://www.memritv.org/Transcript.asp?PI=385. 
Where is the world's conscience? Where are human rights? ¿ $^{8}$ Hier zeigt sich die Notwendigkeit des Kampfes gegen Israel für die Eliten der Arabischen Regime, die in einer Diskussion über interne Menschenrechtsprobleme automatisch auf den äußeren Feind verweisen, um sich so innenpolitisch zu entlasten. 59

Für die Regime stellt sich, einhergehend mit der Menschenrechtsfrage, die Forderung nach Demokratisierung. Der ehemalige libanesische Ministerpräsident Rafiq AlHariri begegnete dieser auf einer Konferenz in Dubai: »Some claim that Islam and democracy cannot coexist. This is completely untrue. Islam coexists with democracy according to rules based on Islamic law.... Islam has its own way of establishing the relation between citizens and officials. It also has its own way concerning transparency and accountability. Islam preceded all the peoples of the world in establishing the principle of shura [consultation] between the ruler and the citizens. «Hier kommt ein Verständnis zum Ausdruck, welches einer institutionalisierten Demokratisierung entgegentritt und sie durch eine Beratschlagung ersetzt. Eine Form einer Demokratie, die unumschränkt auf islamischer Ideologie beruht, ist die Islamische Republik Iran. Zwar ist im Iran eine Form der Gewaltenteilung akzeptiert, aber diese war keine Verpflichtung für die Autoren der Verfassung, da islamische Grundsätze eine derartige Teilung nicht vorsähen. Die Akzeptanz der Gewaltenteilung ist daher lediglich als eine Aufgabenteilung der Regierung zu sehen. Die Mitglieder des Parlaments werden durch ein Zulassungsverfahren vor ihrer Wahl auf ihre Gesinnung durchleuchtet. ${ }^{60}$ Ziel der durch die Verfassung verankerten Herrschaft des Islam im Iran soll es sein, eine fromme Gesellschaft zu schaffen. ${ }^{6}$ Hierbei wird das Parlament durch den Wächterrat kontrolliert, nicht von den islamischen Werten abzuweichen. Dadurch wird eine Islamisierung der Gesellschaft mit einem weitgehenden Herrschaftsanspruch über das Individuum Programm allen staatlichen Handelns. ${ }^{62}$ Im Iran wird also »Göttliches Recht « als weltliche Herrschaft, ohne sich zu säkularisieren, positiviert. Dieses Recht wird dann diktatorisch gegen die eigene Bevölkerung verteidigt. ${ }^{63}$ Mit dem Scheitern der staatlichen so genannten Reformer im Iran tritt dieses diktatorische Element immer weiter in den Vordergrund. ${ }^{64}$

In diesem Kontext erscheint es, wenn Tohidipur schreibt, dass die Umsetzung göttlichen Rechts durch den Wächterrat »nicht die staatliche Gesetzgebung (ersetzt), sondern ihr einen bestimmten Rahmen (setzt) «, ${ }^{65}$ nicht nur verniedlichend, sondern als Rechtfertigung eines diktatorischen Regimes. Tohidipur erkennt hier die Legitimationsideologie des Mullahregimes ohne Kritik an.

\section{c) Reformmoslems: "In the entrance to the auditorium, some people were beaten ${ }^{66}$}

Die bisher dargestellten Positionen benutzen den Bezug auf die Scharia und muslimisches Recht also zur Errichtung einer allumfassenden Ordnung, die sich im Zweifel gegen individuelle Rechte des Subjektes richten. Demgegenüber versuchen aber auch

$58 \mathrm{Ebd}$.

59 Vgl. Rubin (Fn. 33), S. 8 ff.

60 Wahdat-Hagh, Wahied, »Die Islamische Republik Iran«-Die Herrschaft des Islam als eine Spielart des Totalitarismus, Münster 2003, S. 264.

6I Madani, Seyyed Jalaloldin, Hoquqe Assassi dar Jomhuriye Iran, Hakemiat, Welayate Faqih, Qoweye Hakem, II (Verfassungsrecht in der Islamischen Republik Iran, Herrschaftssystem, (Führer), herrschende Gewalt), Band II, Teheran I990, S 5 I.

62 Wahdat-Hagh (Fn. 6o), S. 282 ff.

63 Ebd., S. 250.

64 Rubin (Fn. 33), S. I $17 \mathrm{ff}$.

65 Tohidipur, KJ 2004, S. 3 I0.

66 Der Zuruf eines Studenten der Universität Teheran zu dem so genannten Reformer Khatami, als dieser 
vereinzelte muslimische Intellektuelle, die Scharia als Quelle von Menschenrechten zu nutzen. Es gibt für diese Individuen, die keine gemeinsam übergreifende Ideologie haben, auch keine z. B. den Muslimbrüdern vergleichbare Organisation. Oft werden sie von ihren Herkunftsstaaten behindert oder verfolgt, und sie sind starkem sozialem Druck durch fundamentalistische Bewegungen ausgesetzt. ${ }^{67}$ Auch das Urteil, aus dem Ausland gesteuert zu werden oder ein zionistischer Agent zu sein, schränkt sie in ihrer Arbeit ein.

Diese reformorientierten Moslems sind sich mit den Fundamentalisten in der Analyse der Schwäche und Krise der islamisch geprägten Welt einig. Auch sind sie der Politik des Westens gegenüber oft kritisch, so dass auch sie die doppelten Standards westlicher Wahrnehmung angreifen. Sie streben aber eine historische Einordnung der religiösen Texte in ihre jeweilige Zeit an und negieren somit die Existenz eines ontologischen, in seinem Wesen unabänderlichen islamischen Gesetzes. So veröffentlichte der Tunesische Universitätsdozent Dr. Iqbal Al-Gharbi: »The demand to implement Shari'a today is absurd and ill-intentioned. It indicates its supporters' ignorance both of Islamic history and of the aims of Shari'a. The Prophet Muhammad did not implement Shari'a fully because (Shari'a) was not revealed to him all at once but gradually, over more than 20 years, in response to problems that arose with the organization of the new nation and in response to the challenges (posed) by the Children of Israel in AlMadina... The flaw (in understanding Shari'a) lies in the reliance on a literal reading of the Koran and Sunna, instead of an allegorical and rational reading that sets the best interests of the nation over the texts of its heritage - as did the best of (our) ancient, righteous patriarchs who made us a nation among the advanced nations, that set logic above tradition and placed the best interests of flesh and blood man over the rituals of their forefathers. ${ }^{68}$

Diese Reformmoslems sehen sich drei Fronten gegenüber: die arabischen Regime, die Islamisten und die Globalisierung. Gegen diese Positionen möchten sie aus der Scharia Abwehrrechte konstruieren, indem sie die eigentliche, der Zeit angepasste Scharia, implementieren. So wird versucht, den Islam als Ideologie zu schützen. Diese Position versucht z. B. die Kairoer Dozentin Heba Raouf Ezzat in einem Briefwechsel mit Emran Qureshi von der Harvard Universität deutlich zu machen: »Die Scharia ist der ideale Weg, um Menschenrechte zu verwirklichen. Verstöße gegen Menschenrechte in muslimischen Ländern, deren Regime von westlichen Alliierten unterstützt werden, haben nichts mit der Scharia zu tun. (...) Für (die Islamisten) stellte der Staat das Mittel dar, mit dem die Gesellschaft und die Religion umgeformt werden (konnte). Um eine islamische Renaissance zu erreichen, versuchten sie den Staat zu kontrollieren. (...) ,Scharia bedeutet Weg. Dieser umfasst Glauben und Moralität für ein Individuum, wie auch rechtliche, wirtschaftliche und soziale Rahmenwerke, um das Leben einer Gesellschaft zu regeln. (...) Sie kann weltweit eine egalitäre Kraft für demokratische soziale Gerechtigkeit sein.«69 Dass Heba Raouf Ezzat »kann« statt »ist « benutzt, zeigt, dass ihr bewusst ist, dass die Scharia dies alles heute nicht ist. Hier wird die Scharia also zur Grundlage einer politischen Utopie. Heba Raouf Ezzat fährt fort: Ich »betrachte (die Scharia) als eine Lösung. Diese umfasst die öffentlichen und privaten

unter Buh-Rufen versucht zu erklären, warum die Reformen im Iran erfolgreich sind und warum diese auf keinen Fall durch eine Volksbewegung durchgesetzt werden dürfen, sondern eine staatsinterne Angelegenheit bleiben müssen; www.memritv.org, Clip 40I, http://www.memritv.org/Transcript.asp? $\mathrm{PI}=4 \circ \mathrm{I}$.

67 Milson (Fn. 34), S. I I.

68 Al-Gharbi, Iqbal, Shari'a Must Not Be Implemented Today, www.metransparent.com. Ebenfalls: http:// www.memri.org/bin/articles.cgi?Page=subjects\&Area=reform\&ID=SP79504

69 Briefwechsel zwischen Heba Raouf Ezzat und Emran Qureshi. Der Briefwechsel wurde zwischen Juni und August 2004 geführt und ist erstmals erschienen in der Frankfurter Rundschau vom 4. Oktober 2004, http://www.qantara.de/webcom/show_article.php/_c-323/_nr-8/i.html. 
Sphären und basiert auf zivilen und individuellen Werten. `Zivile Tugend wird in zukünftigen Manifestationen des Islams zentral bleiben.«70

\section{d) Säkularismus: "You are driving us crazy with your fatwas."}

Demgegenüber gibt es Positionen, die derartige Versuche, die Scharia umzudeuten und sie auf ihren »wahren « und humanitären Kern zurück zu führen, als realitätsfremd und als letztlich mit dem Islamismus in bestimmten Argumentationsstrukturen kompatibel zurückweisen. So antwortete Emran Qureshi auf den Brief aus Kairo: »Mir scheint, dass die Realität in unserem Briefwechsel noch nicht ganz angekommen ist. Die heute praktizierte Scharia illustriert Ungerechtigkeit und verneint menschliche Freiheiten. (...) Die existierende Scharia nährt sich vor allem mit Idealen der Salafi und Wahhabi-Ideologie. (...) In meinen Augen ist die Gewalt in erster Linie das Produkt einer erstarrten und globalisierten Salafi- und Dschihadi-Ideologie. Dieser islamistischen Globalisierung müssen wir widerstehen. $\ll^{71}$ Die zentrale Forderung ist die Beschränkung des Islam auf eine persönliche und private Sphäre.

Weitergehende Standpunkte sehen aber darin eine Veränderung, die nach den Ansprüchen aller muslimischen Schulen ein Ende des Islam in seiner heutigen Form bedeuten würde. ${ }^{22}$

Als ein grundsätzliches Problem der Reformmoslems wird gesehen, dass sie im Grundsatz nicht bereit sind, Koran und Sunna in Frage zu stellen und so immer Gott vor die menschliche Vernunft setzen. Auf diese Weise würden sie die Geschichte des Islam und der Araber schönen, da sie in verlogener Weise, aus Furcht gegenüber fremden Einflüssen, nur auf Quellen der eigenen Geschichte verweisen und auch sie Demokratie und Menschenrechte in die islamische Vergangenheit projizieren würden. Schon das Verbot des Religionswechsels, welches durch die islamischen Schriften mit Strafe belegt sei, zeige die grundsätzliche Verachtung islamischer Ideologie für die Rechte und Entscheidungsfreiheit des Einzelnen. Durch den Versuch des Islam, als Rechtsreligion jeden Lebensbereich des Individuums zu regeln, spreche sie dem Menschen immer eine eigene Entscheidungsfreiheit ab. Dieses Konvolut von Regeln habe in der arabischen Welt immer zu Bigotterie und innerer Zerrissenheit geführt. Demgegenüber habe der Islam als dominante Religion in seinen Interpretationen einen totalitären Charakter entwickelt. ${ }^{73} \mathrm{Da}$ Rechte immer nur in der Verbindung mit Pflichten gesehen werden, sei auch der rechtlos, der den Pflichten nicht nachkommt. So neigten muslimische Theoretiker zu einem totalitären Voluntarismus. ${ }^{74}$

Da es keinen institutionalisierten Islam gebe, der wie der Papst ein Deutungsmonopol hätte, wird der Islam von dieser Perspektive als ein beliebig füllbares Gefäß gesehen, welches dazu dient, Kontrolle über Abweichende zu erlangen. ${ }^{75}$ So entgegnete die ägyptische Journalistin Farida Shubaishi auf Zensurforderungen des ehemaligen Präsidenten der Al-Azhar Universität in Kairo bei Liebes- und Sexszenen im ägyptischen Fernsehen: »You want a society that exists only in your mind. You want dogmatic people, whom you create according to your understanding of Islam. (...)You say one thing while other respected Sheiks present a contradictory ruling, and

70 Briefwechsel 2. Brief von Heba Raouf Ezzat (Fn. 70).

7I Briefwechsel 2. Brief von Emran Qureshi (Fn. 70).

72 Warraq, Ibn, Warum ich kein Muslim bin, Berlin 2004, S. 262.

73 Warraq (Fn. 72 ), S. 253 ff.

74 Mayer, Ann Elizabeth, Islam and Human Rights, Boulder i99i, S. 62 f.

75 Dies führt sogar dazu, dass der irakische Ayatollah Sistani ohne weitere Begründung nicht nur Küssen in der Öffentlichkeit, sondern auch das Schachspielen verbietet: www.sistani.org. 


\section{Schlussbemerkung: Wider den Verrat der Intellektuellen}

Tohidipurs Argumentation geht an den Realitäten des Mittleren Ostens vorbei. Er erwähnt in keinem Wort auch nur ein Problem des politischen Islam heute. Reflexartig weist er nur jede Kritik am Islam als Projektion des Westens ab. So übernimmt er Argumentationsmuster von Islamisten, die sich vor Selbstkritik immer durch Verweis auf die Vorurteile des Westens immunisieren. Dass er nicht mit einem Wort die Diskriminierung von Frauen durch die gelebte islamische Ideologie und Praxis erwähnt, und dass auch die Körperstrafen der Scharia unerwähnt bleiben, zeigt, dass er selbst nur ein Bild des Islam in den Mittleren Osten projiziert. Dabei bleibt ein Menschenrechtsverständnis, welches die Anerkennung subjektiver Rechte als notwendige Grundlage sozialer Rechte begreift, auf der Strecke. Durch eine atavistische Zurückweisung von Kritik islamischer Ideologie und muslimischer Gesellschaft ist niemandem geholfen - weder im Westen noch im Mittleren Osten. Tohidipur verliert so zugunsten eines vermeintlichen Antirassismus Universalität und Menschenrechte aus den Augen. Diese falsch verstandene Abwehr eines Eurozentrismus führt - wenn vielleicht auch nicht zwangsläufig - zu einem Verrat der Intellektuellen gegenüber reaktionären Bewegungen, Tyrannen und einer Kultur des Todes.

Die gesellschaftliche Krise der arabischen Welt ist heute derart offenbar, dass auch Kritiker der aktuellen westlichen Politik die Reform- und Revolutionsreife des Mittleren Ostens anerkennen müssen, um zumindest die hausgemachten Probleme in den Griff zu bekommen. Ob hier eine humanisierte Interpretation der Scharia gegenüber einer umfassenden Säkularisierung ausreicht, wird aus hiesiger Perspektive bestritten. Aktuell ist die islamische Reformation des Mittleren Ostens nicht mit der südamerikanischen Befreiungstheologie zu vergleichen, sondern sie ist Ausdruck einer ideologischen Offensive von Islamofaschisten und Diktatoren. Die Frage ist: Inwieweit lässt man sich auf eine feilschende Debatte mit Islamisten ein? Ist es ein Fortschritt, wenn im Iran ein minderjähriges Mädchen für vorehelichen Sex gehenkt statt gesteinigt wird? Ist es ein Fortschritt, ${ }^{77}$ wenn in Nigeria eine Frau, die mit ihrem Ehemann zehnmal am Tag Sex gegen ihren Willen haben musste, nach dem Urteil eines Schariagerichtes sich nur noch viermal am Tag hingeben muss?

Unabhängig von einer Bewertung des amerikanischen Vorgehens im Irak, kann auch von einem neuen Irak aus für den Mittleren Osten eine chancenreiche Perspektive ausgehen. Ein von Irakern durchgeführtes Verfahren gegen Saddam Hussein und seine Schergen, die in Abu Ghraib Schiiten und Kurden über Jahrzehnte still und heimlich zu Tode folterten, könnte zeigen, dass despotische Herrschaft auch im Mittleren Osten nichts Ewiges ist. Die erste demokratische Abwahl einer künftigen irakischen Regierung in den zweiten Wahlen könnte der gesamten muslimischen Welt zeigen, dass König Fahd Unrecht hat. Und der kurdische Nordirak könnte der Nukleus einer föderalen Ordnung sein. Der Islam würde dabei eine genauso große Rolle spielen wie 2004 der Ramadan in der nordirakischen Stadt Sulaimaniyah: ${ }^{78}$ Keine. 